\title{
Improving interpretability of seismic images with directional image partitions and model-based techniques
}

Olga Zdraveva, Joe Zuech, George Zhao, Mohamed Hegazy and Ruoyu Gu

Copyright 2019, SBGf - Sociedade Brasileira de Geofísica

This paper was prepared for presentation during the $16^{\text {th }}$ International Congress of the Brazilian Geophysical Society held in Rio de Janeiro, Brazil, 19-22 August 2019.

Contents of this paper were reviewed by the Technical Committee of the $16^{\text {th }}$ International Congress of the Brazilian Geophysical Society and do not necessarily represent any position of the SBGf, its officers or members. Electronic reproduction or represent any portion of the SBG its oficers or members. Elthout the witten consent of the Brazilian Geophysical Society is prohibited.

\section{Abstract}

Historically, seismic images have always been subject to substantial enhancement to improve their interpretability and/or the quality of reservoir characterization products. We discuss several model-driven techniques for optimizing reverse time and Kirchhoff migration images with directional image partitions. These techniques address various image enhancement challenges common to all geologic settings, such as the presence of strong coherent noise, including migration swings. We, also, introduce a novel effective and pragmatic solution to two frequent problems in areas affected by salt tectonics: the presence of strong residual surface-related multiple energy and converted-wave energy. We ensure that all techniques are scalable and independent of migration algorithm and bandwidth; hence, they could be applied to any size of project and images of any implied frequency content, from high-resolution 3D vertical seismic profile images covering generally small areas to ultra-large exploration images spanning over tens of thousands of $\mathrm{km}^{2}$ of vastly complex and variable geology. We demonstrate their effectiveness on real data examples from the Gulf of Mexico and offshore Brazil, showing significant improvements in the image quality in terms of signal-to-noise ratio, reflector continuity, and amplitude and wavelet consistency, especially in areas of limited illumination.

\section{Introduction}

After a decade of significant advances in seismic acquisition, multiple attenuation, deghosting, signal processing, anisotropic earth model building, and the introduction of reverse time migration (RTM) and full waveform inversion, a critical factor to seismic image interpretability continues to be using image conditioning and enhancement techniques integrated within the imaging workflow that allow for the discrimination of signal and noise to attenuate all undesired events. The combined effects of insufficient illumination, aliasing and the inherent inaccuracy of the earth models yield difficultto-interpret subsalt images even when we use fullazimuth, long-offset seismic data and the most advanced signal processing and imaging techniques available today. In simpler geological settings, image enhancement is still critically important, because the requirement for higher-resolution images, often produced with Kirchhoff depth migration (KDM), normally results in much higher levels of migration swing-related noise.

All image enhancement techniques (both data- and model-based) rely on partial images' ability to separate signal from noise. The model-based techniques, discussed in this work, are guided (implicitly or explicitly) by the geologic structure and require partial images formed by partitioning the energy by direction, achievable in any of the following domains:

1.Pre-migration - e.g., migrating individual shots (Matson et al., 2012) and illumination-guided group of shots or regular shot-patches (Hartman et. al., 2015).

2. During migration at the image collection step e.g., RTM vector image partitions (VIPs) (Xu et al., 2011; O'Briain et al., 2013).

3. Post-migration - e.g., directional image partitions (DIPs) introduced by Nichols et al. (2017) and applicable to any image.

Different methods of partitioning have distinct advantages and disadvantages when solving particular image enhancement challenges. The pre-migration options given above were introduced for RTM and are impractical in many cases, especially for larger-scale projects, although they could be particularly useful on a smaller scale (Zaman et al., 2018). RTM VIPs have a proven record as an interpretation aid (Cogan and Boochoon, 2013; O'Briain et al., 2013; Buker and Maden, 2015) and an image enhancement enabler (Xu et al., 2011; Zhao et al., 2015; Gu et al., 2016), mainly subsalt. DIPs, conversely, are independent of the migration algorithm and allow the ultimate flexibility as they can enable image enhancement for any pre-existing image, irrespective of its origin.

In this work, we discuss three model-based methods using DIPs. First, we outline the advantages and disadvantages of DIPs, contrasting them with wellestablished RTM VIPs. Then, we demonstrate that, introduced by $\mathrm{Gu}$ et al. (2018) dip-guided selective stacking (DGSS) technique can be used successfully even on ultra-large images, including high-resolution KDM and lower-wavenumber RTM images covering more than $70.000 \mathrm{~km}^{2}$ area of extreme complexity. Lastly, we introduce novel and efficient DIPs-enabled, model-based solutions for residual demultiple and converted wave (Cwave) attenuation.

We show successfully applying these model-based image enhancement techniques with DIPs to both RTM and KDM images of variable size and complexity, 
demonstrating the ability to maintain high-frequency, highresolution stratigraphic features, while also enhancing the interpretability of subsalt and steeply dipping events, even in areas of limited illumination.

\section{Directional image partitions and their advantages}

Any seismic image can be decomposed into directional partitions by applying an optimally designed 3D wavenumber filter bank (Nichols et al., 2017). In our experience, using 50 to 100 DIPs adequately samples the $3 \mathrm{D}$ dip hemisphere and allows enough detail and signal and noise separation to enable many image enhancement workflows.

Since their introduction in 2016, DIPs are often preferred over VIPs for the majority of post-migration coherentnoise-attenuation workflows for several reasons:

1) DIPs are applicable to any image, including images from different migration algorithms and/or partial images, such as angle-band stacks.

2) DIPs provide better directional separation of energy compared to VIPs, as the decomposition is explicitly indexed by 3D dip. This is especially true for cases with common use of reciprocity in RTM, such as ocean-bottom nodes and vertical seismic profile (VSP) imaging.

3) Like VIPs, DIPs represent a lossless transformation, i.e., the sum of all DIPs equals exactly the image being partitioned. This aspect is important in preserving relative amplitudes, when required.

4) Model-driven enhancement techniques developed originally for VIPs (Gu et al., 2016) are shown to also work with DIPs (Zdraveva et al., 2017).

5) DIPs are more cost-effective than VIPs, including much simpler and more efficient execution of model-driven workflows. VIPsbased applications require extra computation to evaluate explicitly associated with each VIP 3D dip field, which correspondingly increases the disc storage requirements.

6) DIPs can be used to separate specular events and diffraction energy.

Figure 1a shows an RTM image in an area of obfuscated target illumination, creating difficulties in interpreting the steeply dipping strata on the left of the salt stock, representing a producing reservoir. The presence of steeply dipping events similar to the expected dip at reservoir level (Figure $1 \mathrm{~b}$ ) is evident on both individual VIP (Figure 1c) and DIP (Figure 1e). Figure 1 illustrates how considerable portions of individual DIPs and VIPs will not contribute constructively to the desired target image.

In cases where data-driven image enhancement workflows are important, VIPs still have some advantage, especially when VIP alignment is a requirement for success (Zhao et al., 2015). VIPs are still in use today and, in some cases, data-driven enhancement with VIPs could be followed by model-driven enhancement with
DIPs. In the presence of flexible interactive visualization tools, DIPs could potentially fully replace VIPs as an interpretation aid asset.
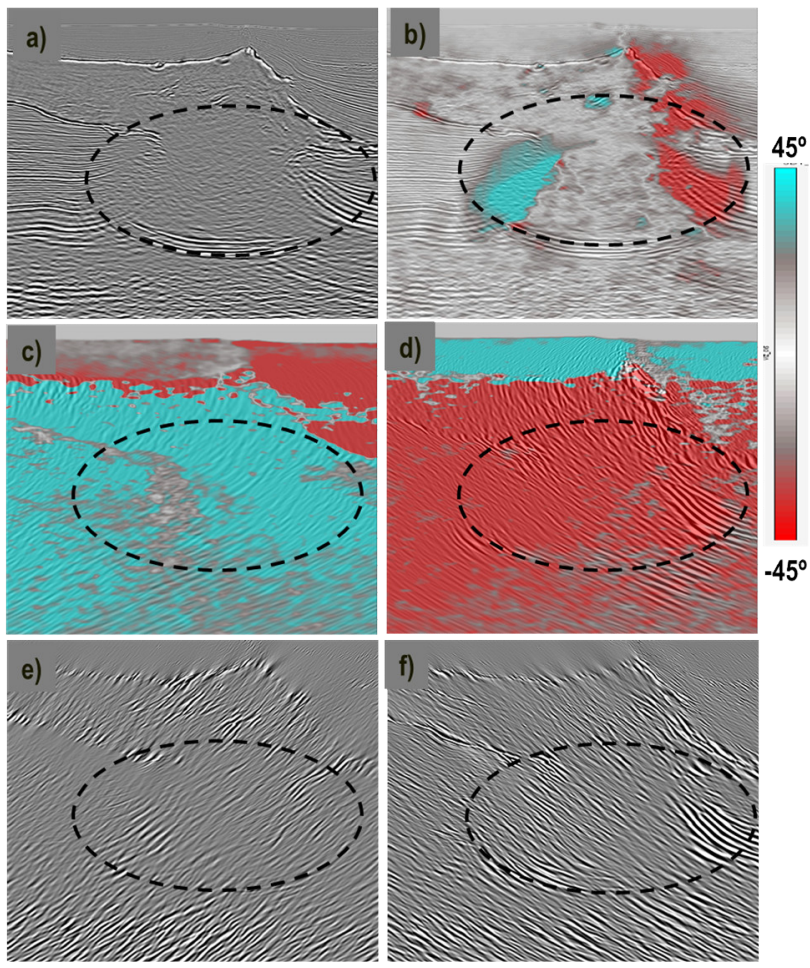

Figure 1 - Raw RTM image in target area: a) without and b) with target inline dip field overlaid; c) and d) two individual RTM VIPs with corresponding inline dip overlaid; e) and f) two invidividual DIPs with $3 D$ dip similar to the target dip in the reservoir area.

\section{Structure-driven image enhancement with DIPs}

Structure-driven image conditioning and enhancement techniques are guided by a geologic model, represented by the target 3D dip field, to discriminate signal from noise on partial images. The target 3D dip field is derived either directly from seismic images or, in cases of low signal-tonoise ratio (SNR) and/or poor illumination, from a set of interpreted horizons, often validated by dip-meter well information. The most commonly used technique employing DIPs today is dip-guided selective stacking (DGSS) (Gu et al., 2018). In this technique, parts of individual DIPs contradicting the target 3D dip field are efficiently down-weighted and/or excluded prior to summing all DIPs to reform the image. This results in successful coherent noise attenuation (Figure 2) and significant improvement of weak events in poorly illuminated areas, including subsalt (Figure 3), based on knowledge of the geologic structure.

In DGSS, the process of down-weighting inconsistent pieces of information on the individual DIPs is flexible enough to allow mild, amplitude-friendly enhancement (Figures $2 \mathrm{~b}$ and $3 \mathrm{~b}$ ) and harsher enhancement revealing extremely weak events not present on the raw image (Figure 3c). Harsher applications are normally intended 
only for structural interpretation, except in the case of 3D VSP imaging where migration swing noise is very strong due to the limited aperture of the physical experiment.
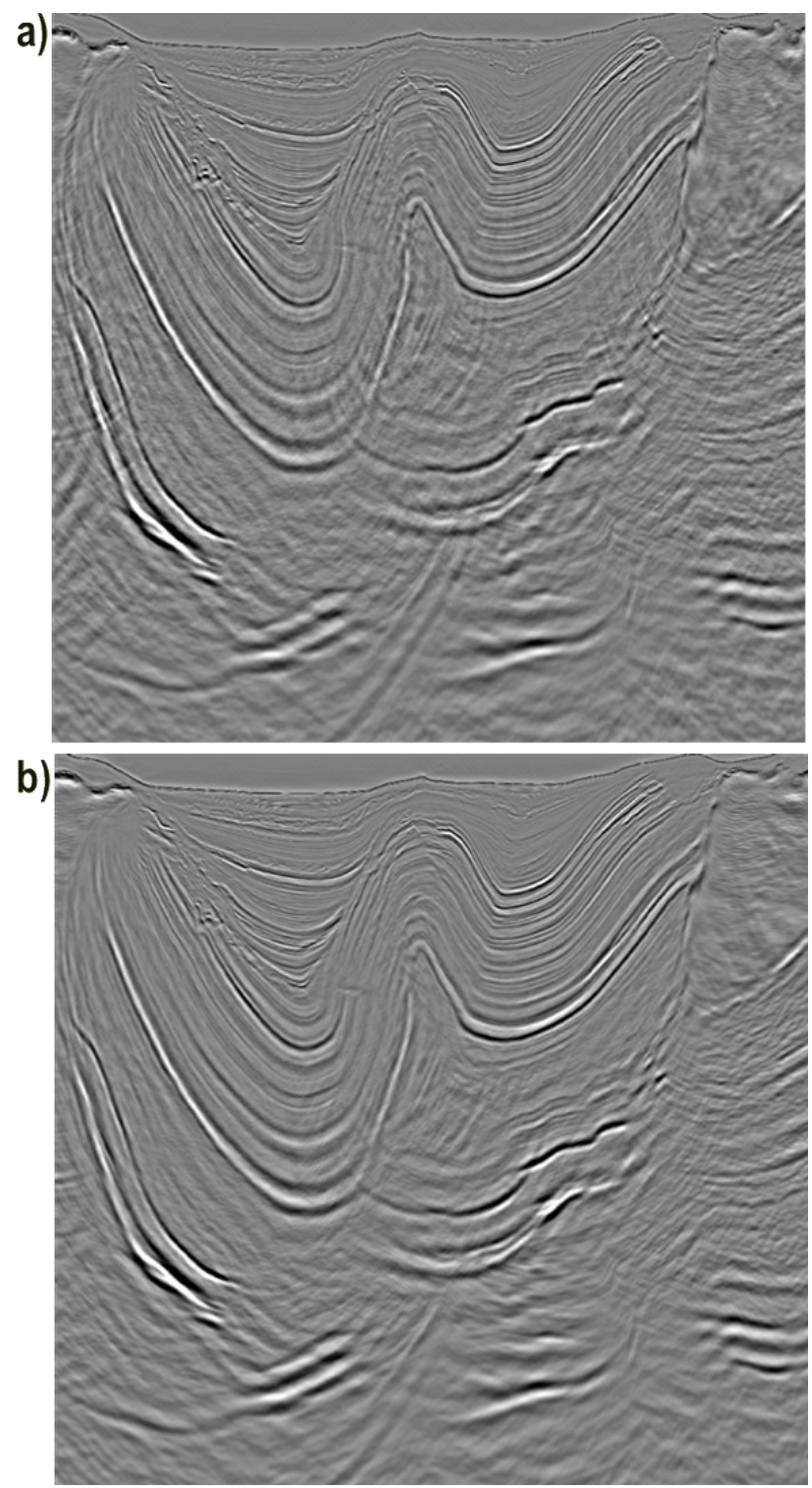

Figure 2 - Large, exploration-scale, high-resolution KDM images: a) raw and b) after mild, general-purpose DGSS successfully attenuated most of the migration swing noise.

\section{Residual multiple attenuation with DIPs}

In modern broadband imaging, intensive efforts to construct a better-deghosted prestack 3D surface-related multiple elimination (SRME) model and optimize leastsquares adaptive subtraction (LSAS) in the pre-migration domain allow successful attenuation of most surfacerelated multiple reflections. Nevertheless, especially in cases of large-scale exploration imaging in areas of extreme geologic complexity, residual multiple energy can still be significant, particularly in subsalt areas.
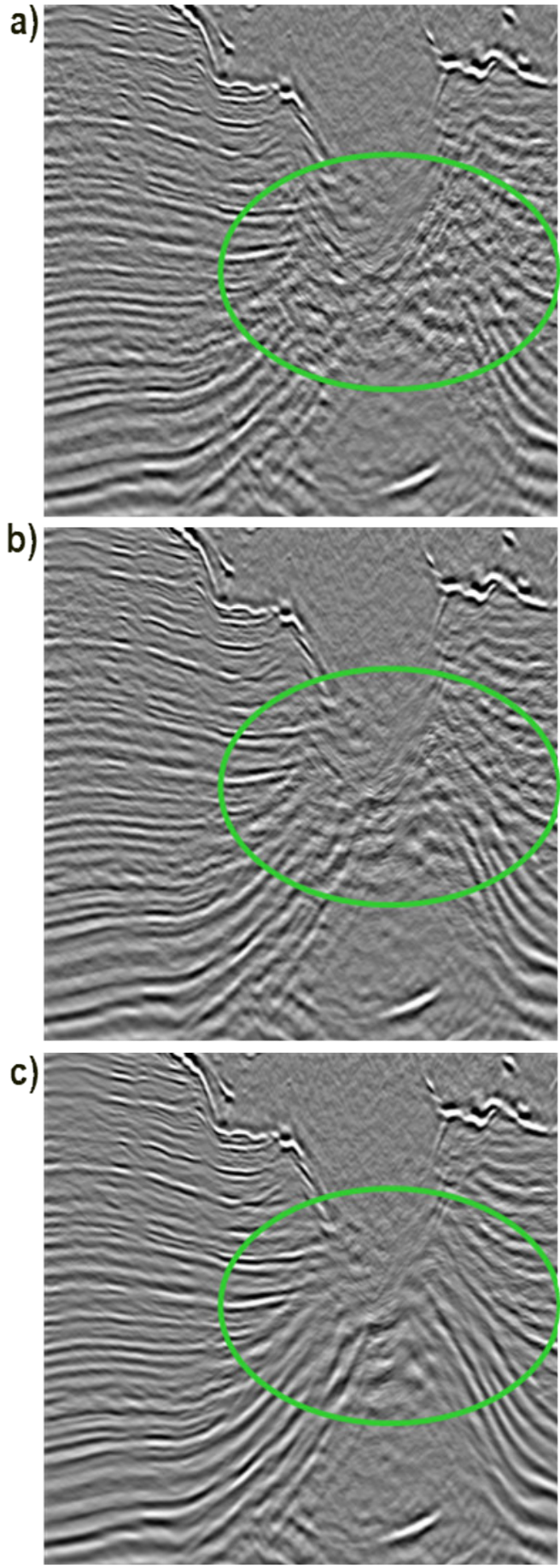

Figure 3 - Reservoir-development scale RTM image around target area: a) raw, b) after mild DGSS, and c) after harsher DGSS, intended for structural interpretation.

Past image-domain approaches to suppress coherent residual multiple noise are centered around a combination of wave-equation multiple modeling, LSAS, dip separation techniques, and/or attribute-based subtraction techniques (Guo et al., 2008; Wang et al., 2010). Another option is to migrate the 3D SRME model and perform residual imagedomain LSAS, but this method can result in artifacts and 
primary attenuation caused by wavelet phase and amplitude mismatches between residual multiple and model.

To effectively attenuate residual multiples without harming primary reflectors we introduce a DIPs-based technique, founded on a cross-correlation weighting scheme, comparing corresponding DIPs from the final RTM image and the RTM image of the 3D SRME model. The final RTM image consists of mainly primaries; hence, the residual multiple noise can be isolated from primary energy using amplitude-based crosscorrelation of the primary image DIPs with the migrated multiple model DIPs to derive a weighting function (equation 1),

$$
W_{i}=D_{i} \theta M_{i} ; k_{n e w}=\frac{\sum_{i=0}^{n} W_{i} * D_{i}}{\sum_{i=0}^{m} W_{i}}
$$

where $D_{i}$ and $M_{i}$ stand for the $i$-th directional partition of primary and multiple images, respectively. $W_{i}$ is the weight derived from $D_{i}$ and $M_{i}$. This weighting function is 3D-variable; hence, it can be used to down-weight residual multiple energy and attenuate it in the combined final image. Inew is the image after residual multiple attenuation.

Figure 4 shows the effectiveness of this method in attenuating residual multiple noise and recovering the underlying primary signal in the southern Gulf of Mexico, which is characterized by extreme geologic complexity. The excessive amount of residual multiple energy in portions of the section (Figures $4 a$ and $4 c$ ) is due to one of the following: (1) the top-of-salt reflection in large portion of the area is $10-20$ times stronger than waterbottom reflection, causing strong interference with primary energy, rendering LSAS less effective globally; (2) the presence of very shallow and rugose top of salt, causing severe primary amplitude loss subsalt at very shallow depths; (3) imperfections in the SRME model due to the use of limited aperture to control cost on ultra-large exploration scale; and (4) it is difficult for LSAS to handle multiple energy effectively in the data domain due to its strong similarity with steeply dipping primary events.

\section{Converted-wave attenuation with DIPs}

It is well-documented that significant mode conversions are created at large velocity contrasts (e.g., salt/sediment interfaces). These salt-related $\mathrm{C}$-waves are mispositioned in the image domain when migrated with $\mathrm{P}$-wave velocity, obfuscating subsalt interpretation and prospecting, especially in areas of poor illumination and low SNR (Ogilvie and Purnell, 1996).

Several studies in recent years identify, model and attenuate C-waves using both pre- and post-migration methods (Huang et al., 2013; Kobylarski et al., 2015; Hegazy et al., 2017). Zaman et al. (2018) showed C-wave attenuation (CWA) results achieved through a LSAS scheme with DIPs, more suitable for smaller-scale projects. We propose an effective and cost-efficient, even on a large scale, CWA workflow through targeted downweighting DIPs containing C-wave energy. It is centered around forward modeling the $\mathrm{C}$-wave base of salt (BOS), accomplished by map demigrating the primary BOS surface using a velocity model with S-velocity inside salt, then map remigrating using $P$-velocity. This simulates a PSSP mode conversion and gives us a good approximation of the apparent 3D dip of a generalized Cwave BOS surface. Figure 5 visually depicts the $\mathrm{C}$-wave surface derivation steps.
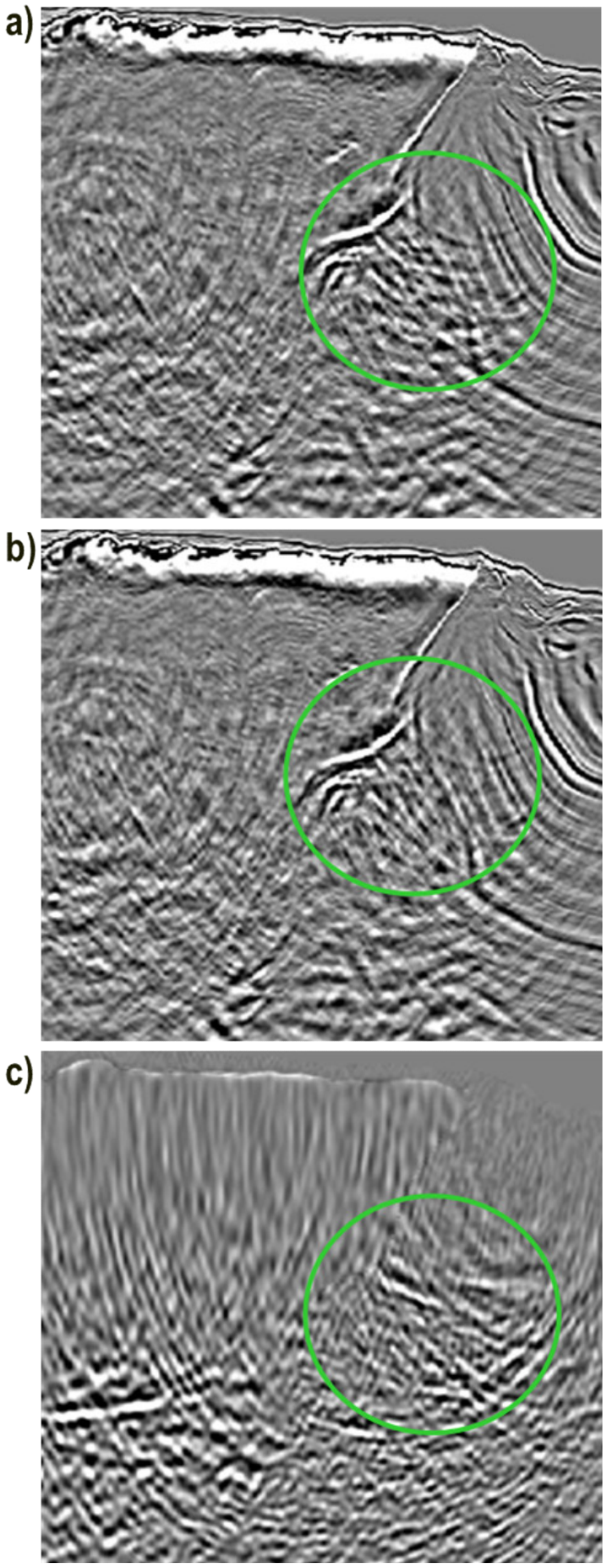

Figure 4 - RTM image: a) before, and b) after residual multiple attenuation with DIPS; and c) $3 D$ SRME multiple model. 


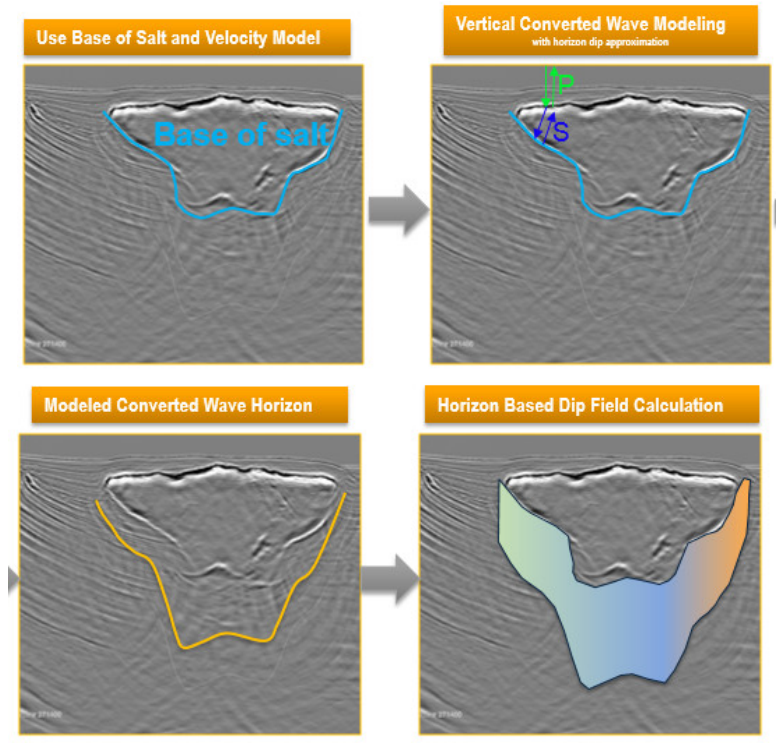

Figure 5 - C-wave BOS surface modeling and VOI-based dip estimation workflow.

The next step involves running two 3D dip estimations: (1) purely image-based to estimate the structural 3D dip; (2) hybrid with the image in conjunction with a horizon-guided volume of interest $(\mathrm{VOI})$, bounded by the BOS and the Cwave BOS. The two dip estimations will be identical outside of the VOI and can differ inside the VOI.

We employ a dissimilarity measure between the two $3 D$ dip fields to create a smooth operating mask for the CWA process. This acts as a form of signal protection, as we do not allow CWA to operate when the C-wave 3D apparent dip is too close to the structural 3D dip, which allows us to apply the process survey-wide without the risk of harming prospective subsalt primary reflectors.

The image containing both primary reflections and Cwave noise is then decomposed into DIPs. As attempting to subtract modelled C-wave energy can lead to spurious artefacts and/or signal attenuation, we instead employ a weighting scheme described by equation 2 ,

$$
W_{i}=1-(\hat{\mathbf{I}} \cdot \bullet \hat{\mathbf{C}})
$$

where $\tilde{L}_{i}$ is the unit vector of the $3 \mathrm{D}$ dip corresponding to the $i$-th DIP and $\hat{C}$ is the unit vector of the apparent 3D dip of the modelled C-waves. The scalar weight $\left(W_{i}\right)$ applied to each DIP is one minus the dot product of $\hat{\boldsymbol{i}} \boldsymbol{i}$ and $\tilde{\boldsymbol{C}}$. It is worth noting that this weighting scheme is independent of seismic amplitude, differing from the crosscorrelation scheme used during the model-based residual multiple attenuation. This fact allows us to reuse the weights derived during RTM CWA for the KDM CWA without rerunning the computation. Figure 6 shows the CWA result on the final high-resolution KDM image. The Cwaves dominate the subsalt image prior to CWA. PostCWA, C-waves are well-attenuated, revealing clearer subsalt reflectors that truncate at the BOS.
Attenuating C-wave energy is particularly important in reservoir-development imaging. The CWA method we introduced is complementary to any other data or modeldriven techniques we may choose to use on any project.

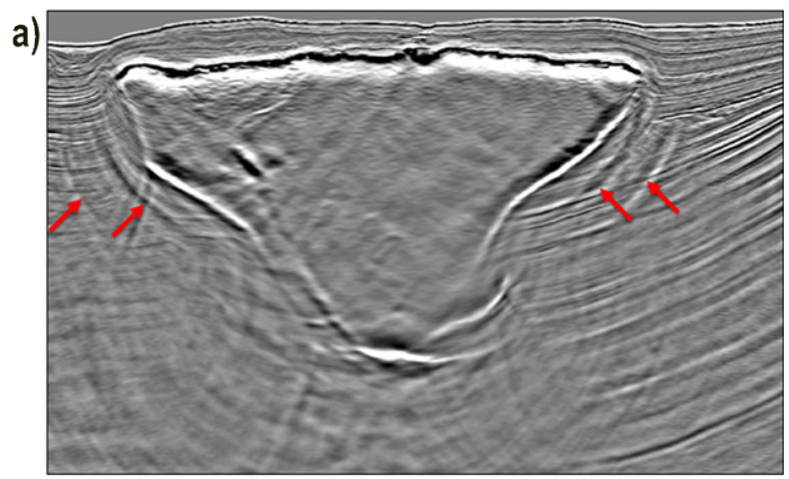

b)

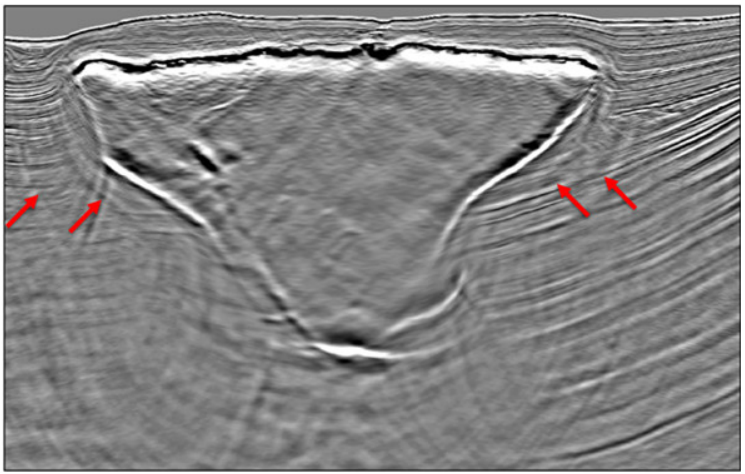

Figure 6 - KDM images: a) before, and b) after applying CWA. Arrows indicate strong $C$-waves that are attenuated after CWA.

\section{Conclusions}

Model-based image enhancement methods with DIPs detailed in this work, successfully leverage structural geology information to robustly improve the interpretability of seismic images of different scale, complexity, and origin. We showed that in the presence of a reliable and representative geologic structure model, DGSS is equally effective in mild overall image enhancement, substantially attenuating migration swing noise in broadband KDM images, and in dramatic changes in subsalt interpretability, revealing subsalt events that are hidden under noise in raw RTM images. We presented examples of successfully attenuating residual multiple and C-wave energy, complementing the application of dip-guided selective stacking on an ultra-large scale. All examples exhibit significant improvements in signal-to-noise ratio as well as general interpretability of events and known subsalt targets.

\section{Acknowledgments}

We thank WesternGeco Multiclient for the permission to show the data used this case study and many colleagues in WesternGeco for their contributions. 


\section{References}

BUKER, F., MADEN, S. Application of reverse time migration vector image partitions to narrow azimuth data acquired in a faulted region. $77^{\text {th }}$ EAGE Conference \& Exhibition, Extended Abstracts, 2015.

GU, R., ZDRAVEVA, O., HEGAZY, M., BUZZELL, S. Interpretation-guided image enhancement using reverse time migration vector image partitions. $86^{\text {th }}$ Annual International Meeting, SEG, Expanded Abstracts, 43414345, 2016.

GU, R., HEGAZY, M., ZDRAVEVA, O. Geologic structurebased image enhancement with directional image partitions. $80^{\text {th }}$ EAGE Conference \& Exhibition, Extended Abstracts, 2018.

GUO, M., KIM, Y., SEN, S., XU, J., XIE, J., WANG, B. A new multiple subtraction method using the attributes of predicted multiples. $78^{\text {th }}$ SEG Annual International Meeting, SEG, Expanded Abstracts, 2451-2455, 2008.

HARTMAN, K., CHAKRABORTY, S., NOLTE, B., GOU, W., SUN, Q., CHAZALNOEL, N. Understanding and improving the subsalt image at Thunder Horse, Gulf of Mexico. $85^{\text {th }}$ Annual International Meeting, SEG, Expanded Abstracts, 4028-4032, 2015.

HEGAZY, M., STEWART, A., HYDAL, S., MALAVE, K., MATARACIOGLU, O. Salt-related converted-wave attenuation. $87^{\text {th }}$ Annual International Meeting, SEG, Expanded Abstracts, 4609-4613, 2017.

HUANG, Y., GOU, W., LEBLANC, O., LI, Y., JI, S., HUANG, Y. Study and application of salt-related converted waves in imaging. $83^{\text {rd }}$ Annual International Meeting, SEG, Expanded Abstracts, 3851-3855, 2013.

KOBYLARSKI, M, TYSON, C., LONG, S. Suppressing sub-salt converted mode interference: An example from deep-water Angola. 85 ${ }^{\text {th }}$ Annual International Meeting, SEG, Expanded Abstracts, 4044-4048, 2015.

MATSON, K., CLIPPARD, J., CATTERMOLE, P. Signal enhancement using diversity stacking for reverse time migration (DeSSeRT). 82 ${ }^{\text {nd }}$ Annual International Meeting, SEG, Expanded Abstracts, 1-5, 2012.

NICHOLS, D., RUSE, A., HYDAL, S. Separating diffractions and specular reflections after imaging. $79^{\text {th }}$ EAGE Conference \& Exhibition, Extended Abstracts, B105, 2017.

O'BRIAIN, M., SMITH, D., MONTOYA, C., BURGESS, B., KOZA, S., ZDRAVEVA, O., ISHAK, M., ALWON, S., KING, R., NIKOLENKO, D., VAUTIER, S. Improved subsalt imaging and salt interpretation by RTM scenario testing and image partitioning. $83^{\text {rd }}$ Annual International Meeting, SEG, Expanded Abstracts, 3856-3860, 2013.
OGILVIE, J. S., PURNELL, G. W. Effects of salt-related mode conversions on subsalt prospecting. Geophysics, 61, 331-348, 1996.

WANG, B., MASON, C., GUO, M., CAI, J., GAJAWADA, S., EPILI, D. Interactive demultiple in the post-migration depth domain. $80^{\text {th }}$ Annual International Meeting, SEG, Expanded Abstracts, 3436-3440, 2010.

XU, Q., LI, Y., YU, X., HUANG, Y. Reverse time migration using vector offset output to improve subsalt imaging $-A$ case study at the Walker Ridge GOM. 81'st Annual International Meeting, SEG, Expanded Abstracts, 32693274, 2011.

ZAMAN, S. O., SARAGOUSSI, E., SOLANO, G. A., ZDRAVEVA, O., HERNANDEZ, V. R. B., LI, Q., CHAKRABORTY, S., JOY, C., HARTMAN, K., REITZ, A. Image Enhancements for Subsalt targets; Thunder Horse case study. 88 ${ }^{\text {th }}$ Annual International Meeting, SEG, Expanded Abstracts, 4573-4577, 2018.

ZDRAVEVA, O., GU, R., HEGAZY, M. Improving interpretability of subsalt targets by geologic structurebased image-enhancement with different image partitions. APGCE 2017 Conference and Exhibition, Extended Abstracts, 2017.

ZHAO, C., ZDRAVEVA, O., GONZALEZ, A., KING, R., GU, R. Improving interpretability of subsalt images by image conditioning and enhancement with RTM vector image partitions. $77^{\text {th }}$ EAGE Conference \& Exhibition, Extended Abstracts, N107-14, 2015. 